\title{
MOLECULAR BASIS OF DIFFERENCE IN ALCOHOL METABOLISM BETWEEN ORIENTALS AND CAUCASIANS
}

\author{
(The Japan Society of Human Genetics Award Lecture)
}

\author{
Akira YosHIDA \\ Department of Biochemical Genetics, City of Hope Research \\ Institute, Duarte, California, U.S.A.
}

\begin{abstract}
Summary The human cytosol alcohol dehydrogenase(ADH) controlled by $\mathrm{ADH}_{2}$ locus is known to be polymorphic. Most Caucasians have the usual enzyme consisting of the usual $\beta_{1}$ subunit, while nearly $90 \%$ of Orientals have the atypical $\beta_{2}$ subunit. At the physiological $\mathrm{pH}$, the cytosol enzyme activity of the atypical liver is much higher than that of the typical liver. It has been suggested that a high frequency of acute alcohol intoxication among Orientals could be related to the rapid accumulation of acetaldehyde due to the atypical ADH. Human liver aldehyde dehydrogenase (ALDH) is also polymorphic. Virtually all Caucasians have two major isozymes, i.e., ALDH-1 and ALDH-2, while about $50 \%$ of Orientals have only ALDH-1, missing ALDH-2 component. Since ALDH-2 has high affinity to acetaldehyde, the high incidence of alcohol sensitivity in Orientals could be related to the accumulation of acetaldehyde due to the lack of ALDH-2 component. The usual ADH homodimer $\beta_{1} \beta_{1}$ and the atypical homodimer $\beta_{2} \beta_{2}$ were purified to homogeneity. The specific activity of the atypical enzyme was nearly three orders of magnitude higher than the usual enzyme at the physiological $\mathrm{pH}$. The usual enzyme was rapidly inactivated by iodoacetate, indicating an existence of the "active site cysteine" in the molecule. In contrast, the atypical enzyme was resistant against the iodoacetate inactivation. Peptide mapping analysis revealed that the active site cysteine in the usual $\beta_{1}$ subunit is replaced by histidine in the atypical $\beta_{2}$ subunit. In the usual $\beta_{1} \beta_{1}$ enzyme, like horse enzyme, the catalytic $\mathrm{Zn}$ is expected to link to the sensitive cysteine at position 47 , histidine at position 67 , and cysteine at presumably position 174 , thus making up the active site. In contrast, the active site of the atypical $\beta_{2} \beta_{2}$ enzyme is expected to consist of the catalytic $\mathrm{Zn}$ linked to histidine at position 47 , histidine at position 67 , and cysteine at position around 174 . The functional abnormalities of the atypical enzyme can be correlated to. its molecular abnormality.
\end{abstract}


ALDH-1 consists of 4 subunits of molecular weight of 56,500 and ALDH2 consists of 4 subunits of molecular weight of 52,500. These isozymes do not share any common subunit. Examination of liver extracts by two dimensional crossed immunoelectrophoresis revealed that an atypical Japanese liver, which had no ALDH-2 isozyme, contained an enzymatically inactive but immunologically cross reactive material corresponding to ALDH-2 isozyme, besides the active ALDH-1 isozyme. Therefore, the absence of ALDH-2 isozyme in atypical Orientals is not due to regulatory mutation, gene deletion, or nonsense mutation, but it must be due to a structural mutation in a gene for ALDH-2 locus, resulting in synthesis of enzymatically inactive abnormal protein. Based on the available data, a possible correlation between the enzyme abnormalities and the alcohol sensitivity was discussed.

\section{INTRODUCTION}

Individual and racial differences in alcohol intoxication have been reported by many investigators. "Alcohol sensitive" persons exhibit rapid facial flushing, elevation of skin temperature and increase in pulse rate, when they drink $0.3-0.5 \mathrm{ml}$ of ethanol $/ \mathrm{kg}$ body weight. The sensitivity to alcohol is far more commonly observed in individuals of Mongoloid origin (Japanese, Chinese, Korean, and American Indian) than in Caucasians. According to Wolff $(1972,1973)$, more than $80 \%$ of Orientals are alcohol sensitive, while only $5 \%$ of Caucasians respond abnormally after alcohol administration. Somewhat lower frequencies of alcohol sensitivity in Orientals were reported by other investigators. The racial difference is thought to be related to genetic differences in the enzymes involved in alcohol metabolism.

Alcohol is metabolized mainly by liver alcohol dehydrogenase (ADH), and aldehyde thus produced is further oxidized by aldehyde dehydrogenase (ALDH). The cause of "acute alcohol intoxication" symptom is mainly the action of acetaldehyde which stimulates the release of epinephrine, norepinephrine and other agents (Duritz and Truitt, 1966; Borg and Kvande, 1981). The human liver contains several cytosol ADH and microsomal ADH. One of the major cytosol ADH isozymes, controlled by $\mathrm{ADH}_{2}$ locus, differs between Caucasians and Orientals. Most Caucasians have the usual $\mathrm{ADH}_{2}{ }^{1}$ enzyme, while nearly $90 \%$ of Orientals have the atypical $\mathrm{ADH}_{2}{ }^{2}$ enzyme which exhibits much higher enzyme activity than the usual enzyme, particularly at the physiological $\mathrm{pH}$ (Stamatoyannopoulos et al., 1975). Thus Stamatoyannopoulos et al. (1975) suggested that the racial difference in alcohol sensitivity could be due to the rapid acetaldehyde formation by the superactive atypical $\mathrm{ADH}$ in many Orientals.

Liver aldehyde dehydrogenase components also differ in Caucasians and Orientals. Virtually all Caucasians have two major isozymes, i.e., ALDH-1 and ALDH2, while approximately $50 \%$ of Orientals have only ALDH-1 isozyme, missing 
ALDH-2 isozyme (Goedde et al., 1979a, Teng, 1981). Since ALDH-2 has a high affinity to acetaldehyde, an absence of this isozyme could induce elevation of acetaldehyde concentration in the atypical subjects. Goedde et al. (1979a) proposed that an absence of ALDH-2 might be related to the high incidence of alcohol sensitivity among Orientals.

This paper describes a) functional and molecular differences between the usual and atypical $\mathrm{ADH}_{2}$, b) functional differences between ALDH-1 and ALDH-2 isozymes, and genetic mechanism of the absence of ALDH-2 isozyme in many Orientals, and c) possible correlation between the alcohol sensitivity and the enzyme abnormalities.

\section{RESULTS AND DISCUSSION}

a) Alcohol dehydrogenase. Human cytosol alcohol dehydrogenase contains several homo- and heterodimers formed by the association of three types of subunits $\alpha, \beta$, and $\gamma$, which are governed by the three separate structural loci, $A D H_{1}$, $A D H_{2}$, and $A D H_{3}$, respectively (Fig. 1) (Smith et al., 1971, 1972, 1973). The Oriental atypical enzyme contains an atypical $\beta_{2}$ subunit, i.e., a product of an atypical $A D H_{2}$ gene. Genetic polymorphism is found to exist also in $A D H_{3}$ loci (Smith et al., 1972), while locus $A D H_{1}$ is probably monomorphic, poorly expressed in adult and more strongly expressed in infant (Smith et al., 1971). The usual homodimer $\beta_{1} \beta_{1}$ and the atypical homodimer $\beta_{2} \beta_{2}$ isozymes were purified to homogeneity from an usual Caucasian liver and an atypical Japanese liver (Figs. 2 and 3). The usual $\beta_{1} \beta_{1}$ is more active at $\mathrm{pH} 10.5$, while the atypical $\beta_{2} \beta_{2}$ exhibits maximum activity at $\mathrm{pH} 8.0$. The specific activity ( $V_{\max } / \mathrm{mg}$ enzyme) of the atypical enzyme was several times higher at $\mathrm{pH} 10$, and nearly three orders of magnitude higher at the physiological $\mathrm{pH}$, than that of the usual enzyme. $K_{\mathrm{m}}$ values for ethanol, NAD, acetaldehyde, and NADH were several times higher in the atypical enzyme than in the usual enzyme (Table 1). The usual enzyme was rapidly inactivated by iodoacetate, indicating the existence of an "active site cysteine" in the molecule. In contrast, the atypical enzyme was resistant to iodoacetate inactivation (Fig. 4).

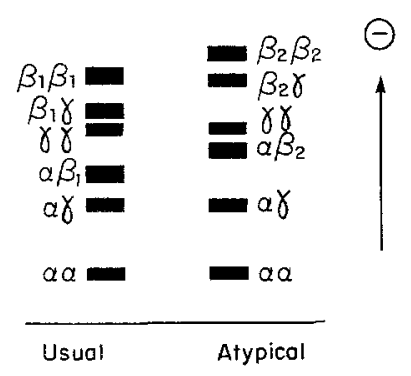

Fig. 1. Schematic presentation of starch gel electrophoresis patterns of the usual and atypical human alcohol dehydrogenase isozymes. 


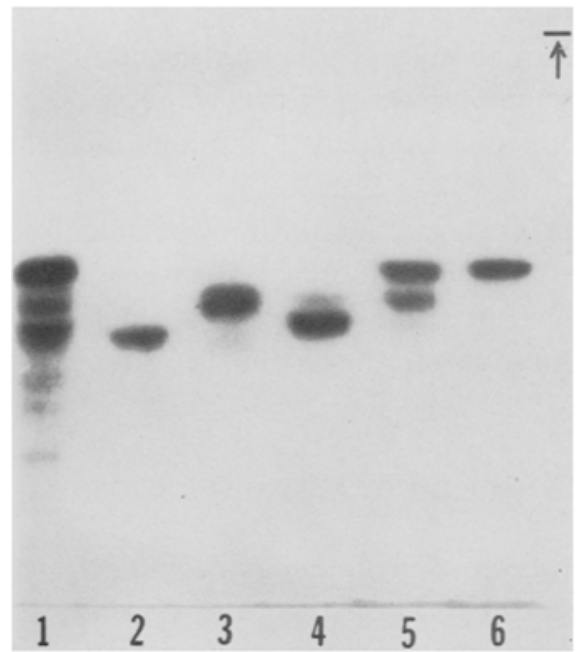

Fig. 2. Starch gel electrophoresis patterns of the usual human alcohol dehydrogenase isozymes. Starch gel electrophoresis was carried out at pH 8.6, and the enzyme was stained for activity. 1: Crude extract of liver with usual phenotype; 2: First isozyme peak eluted from CM-cellulose; 3: Second isozyme peak eluted from CM-cellulose; 4: Third isozyme peak eluted from CM-cellulose; 5: Fourth isozyme peak eluted from CM-cellulose; 6: Fifth isozyme peak eluted from CMcellulose. The fifth isozyme peak contains only $\beta_{1} \beta_{1}$ component. Details were reported by Yoshida et al. (1981).

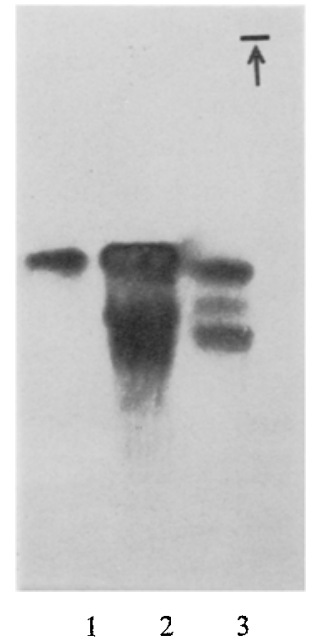

Fig. 3. Starch gel electrophoresis patterns of the usual and atypical human alcohol dehydrogenase isozymes. 1 : Atypical $\beta_{2} \beta_{2}$ component isolated by CM-cellulose chromatography; 2: Crude extract of liver with atypical phenotype; 3 : Crude extract of liver with usual phenotype. Details were reported by Yoshida et al. (1981). 
Table 1. Kinetic properties of usual and atypical alcohol dehydrogenase.

\begin{tabular}{|c|c|c|c|c|c|}
\hline & & \multicolumn{2}{|c|}{ Usual $\beta_{1} \beta_{1}$} & \multicolumn{2}{|c|}{ Atypical $\beta_{2} \beta_{2}$} \\
\hline & & $\mathrm{pH} 8.5$ & $\mathrm{pH} 10.0$ & pH 8.5 & $\mathrm{pH} 10.0$ \\
\hline \multirow{2}{*}{$\begin{array}{l}\text { Specific activity } \\
\text { (units/mg) }\end{array}$} & Ethanol $\longrightarrow$ Aldehyde & 0.39 & 0.8 & 32 & 5.0 \\
\hline & Aldehyde $\longrightarrow$ Ethanol & 1.8 & 0.48 & 1.6 & 12.5 \\
\hline \multirow[t]{4}{*}{$K_{\mathrm{m}}(\mathrm{mM})$} & Ethanol & 0.37 & 0.78 & 2. 1 & 5.0 \\
\hline & NAD & 0.039 & 0.064 & 0.15 & 0.69 \\
\hline & Aldehyde & 0.94 & 1. 14 & 1. 24 & 19.2 \\
\hline & NADH & 0.0097 & 0.014 & 0.078 & 0.17 \\
\hline
\end{tabular}

The enzyme activity was assayed in $30 \mathrm{~mm}$ glycine/ $\mathrm{NaOH}$ buffer at $25^{\circ} \mathrm{C}$. The reaction mixture for the forward assay (ethanol $\rightarrow$ acetaldehyde) contained $1 \mathrm{mM}$ NAD and various concentrations of ethanol, or $10 \mathrm{~mm}$ ethanol and various concentrations of NAD. The reaction mixture for the backward assay (acetaldehyde $\rightarrow$ ethanol) contained $0.5 \mathrm{mM} \mathrm{NADH}$ and various concentrations of acetaldehyde, or $20 \mathrm{~mm}$ acetaldehyde and various concentrations of NADH.

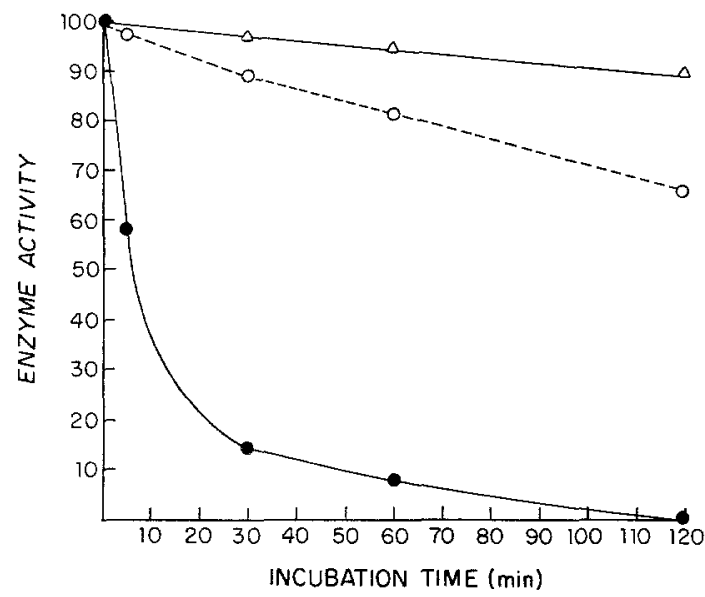

Fig. 4. Inactivation of usual $\beta_{1} \beta_{1}$ and atypical $\beta_{2} \beta_{2}$ enzymes by iodoacetate. The purified enzymes were incubated in $0.02 \mathrm{M}$ Tris chloride, pH 7.5 , at $25^{\circ} \mathrm{C}$. The enzyme activity is expressed taking the original activity as $100 . \Delta$, usual $\beta_{1} \beta_{1}$ enzyme without iodoacetate; $\bullet$, usual $\beta_{1} \beta_{1}$ enzyme with $0.5 \mathrm{~mm}$ iodoacetate; 0 , atypical $\beta_{2} \beta_{2}$ enzyme with $0.5 \mathrm{~mm}$ iodoacetate. Details were reported by Yoshida et al. (1981).

For structural study, the usual and atypical enzymes were $S$-carboxymethylated or $S$-aminoethylated, and then digested by trypsin. The tryptic digests were separated by two dimensional paper chromatography-electrophoresis (Fig. 5). The determination of amino acid compositions and sequences of the peptides implicated in the 


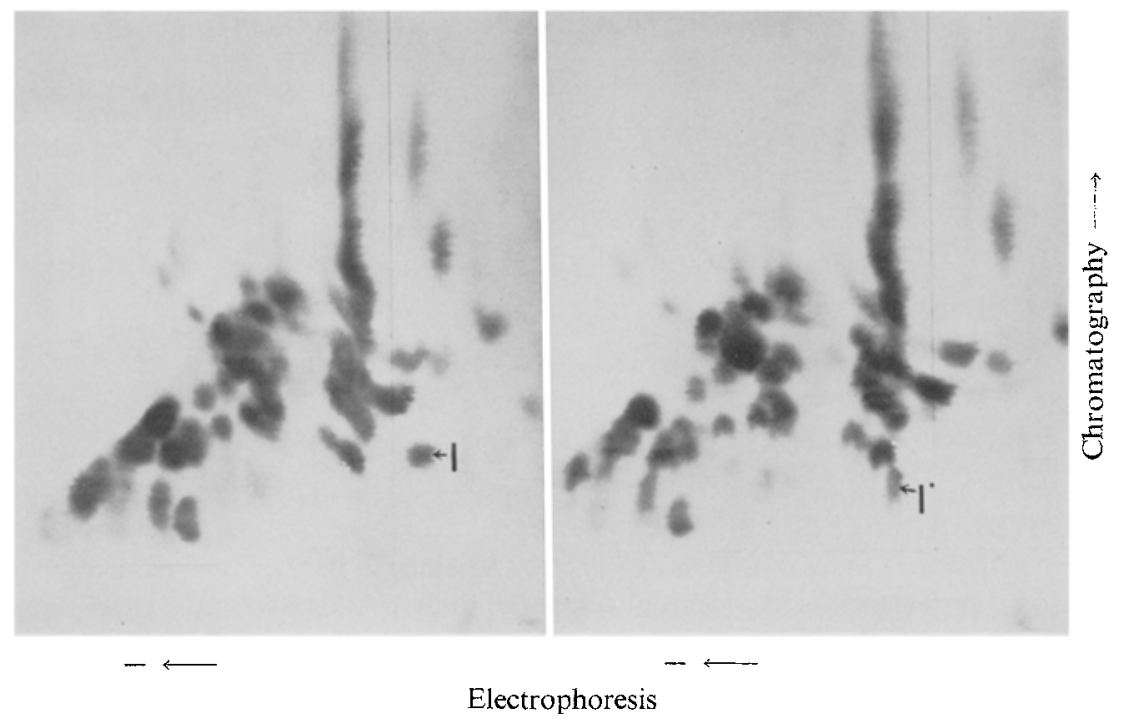

Fig. 5. Peptide maps of tryptic digests of usual $\beta_{1} \beta_{1}$ and atypical $\beta_{2} \beta_{2}$ enzymes. It is shown that the peptide marked I in $\beta_{1} \beta_{1}$ (left) is replaced by the peptide marked $\mathrm{I}^{\prime}$ in $\beta_{2} \beta_{2}$ (right). Details were reported by Yoshida et al. (1981).

Horse Fnzyme

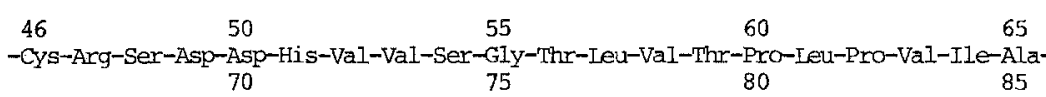

Gly-His-Glu-Ala-Ala-Gly-Ile-Val-Glu-Ser-Tle-Gly-Glu-Gly-Val-Thr-Thr-Val-Arg-Pro-Gly-Asp-Lys-

Human $B_{1} \beta_{1}$

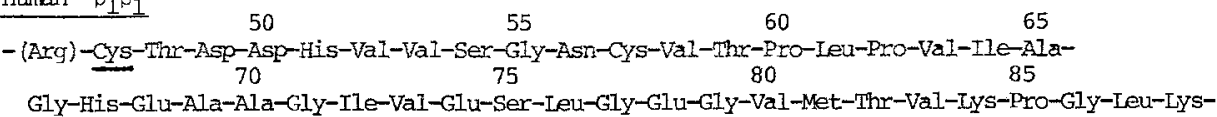

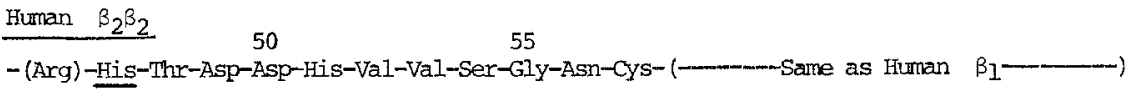

Fuman $\alpha$ and/or $Y$

-Cys-Arg-

Fig. 6. Structure of "active site" of alcohol dehydrogenases. The positions of the amino acid substitutions are underlined. The structures of usual human $\beta_{1} \beta_{1}$ and atypical human $\beta_{2} \beta_{2}$ are based on the data of Yoshida et al. (1981). The structure of human $\alpha$ and/or $\gamma$ is based on the report of Jörnvall and Pictruszko (1972). The structure of the horse enzyme is based on the sequence data of Jörnvall (1970).

structural difference revealed that the active site cysteine in the usual $\beta_{1}$ subunit is replaced by histidine in the atypical $\beta_{2}$ subunit (Fig. 6).

The best studied ADH is the horse cytosol enzyme. The complete amino acid sequence of horse liver ADH has been reported (Jörnvall, 1970), and its three di- 
mensional structure was proposed based on X-ray crystallography (Eklund et al., 1976). A "catalytic" Zn links to Cys at position 46, His at position 67, and Cys at position 174, constituting the active site of the horse enzyme (Eklund et al., 1976). Cys at position 46 is rapidly $S$-carboxymethylated by iodoacetate and the enzyme becomes inactive (Jörnvall, 1970; Dahl and McKinley-McKee, 1980). A remarkable structural homology exists between the horse and human enzyme in this part of the molecule (Fig. 6). In the usual human $\beta_{1} \beta_{1}$ enzyme, the catalytic $\mathrm{Zn}$ is expected to link to Cys at position 47, His at position 67, and Cys at position 174, although the exact position of this particular Cys is not yet determined. Thus, the structure of the active site of the human $\beta_{1} \beta_{1}$ enzyme should be essentially identical with that of the horse enzyme. In contrast, in the atypical $\beta_{2} \beta_{2}$ enzyme, the catalytic $\mathrm{Zn}$ should link to His 47 , His 67 , and Cys 174 . The resistance of the atypical enzyme to inactivation by iodoacetate is a direct consequence of the absence of a sensitive Cys in the active site of the atypical enzyme.

The precise mechanism of alcohol dehydrogenase is not yet fully disclosed, and the explanation of the differences in kinetic properties (i.e., $\mathrm{pH}$ optima, specific activity, $K_{\mathrm{m}}$ values for the substrates and co-enzyme) due to the structural change (i.e., Cys $\rightarrow$ His at position 47) cannot be given at the present time. Future comparative kinetic and X-ray crystallographic studies of the usual and the atypical enzymes would lead us to understand the mechanism of alcohol dehydrogenase action in more detail. Horse ADH has a Cys-Arg linkage at position 46-47, and it has been reported that the human enzyme also has a Cys-Arg at the corresponding position (Jörnvall and Pietruszko, 1972). However, the usual $\beta_{1} \beta_{1}$ has no such linkage; instead, Arg-Cys exists at the active site. The previous analysis was made using a mixture of isozymes, and the Cys-Arg could presumably originate from $\alpha$ and/or $\gamma$ subunits. The yield of a tryptic peptide containing this linkage was indeed very low (Jörnvall and Pietruszko, 1972). Judging from the structure of the horse enzyme, the original form of liver ADH has Cys-Arg at the active site, and the $\beta$ subunit is considered to be evolved from this form. The substitution Cys $\rightarrow$ Arg is compatible with a single base replacement. Two independent substitutions, i.e., Cys $\rightarrow$ Arg at position 46 , and $\mathrm{Arg} \rightarrow$ Cys at position 47 , are required for producing $\beta_{1}$ from a putative original form. If a Cys $\rightarrow$ Arg substitution occurred first, the enzyme would be expected to lose activity, since Cys is essential for $\mathrm{Zn}$ binding and Arg has no metal chelating activity. Therefore, $\beta_{1}$ is likely to originate by the substitution Arg $\rightarrow$ Cys at position 47 , and a subsequent Cys $\rightarrow$ Arg substitution at position 46, thus always preserving its catalytic activity during the evolutionary process. In the case of atypical $\beta_{2}$, an Arg $\rightarrow$ His substitution at position 47 and a subsequent $\mathrm{Cys} \rightarrow$ Arg substitution at position 46 would take place (Scheme 1 in Fig. 7). An alternative evolutionary process could be ancestral from $\rightarrow \beta_{1} \rightarrow \beta_{2}$, or ancestral from $\rightarrow \beta_{2} \rightarrow \beta_{1}$ (Schemes 2 and 3 in Fig. 7). This possibility is less likely, since the occurrence of a catalytically inactive form of enzyme is inevitable in the process. Whatever the evolutional mechanism had been, a minimum of two steps of base substitution is required to 


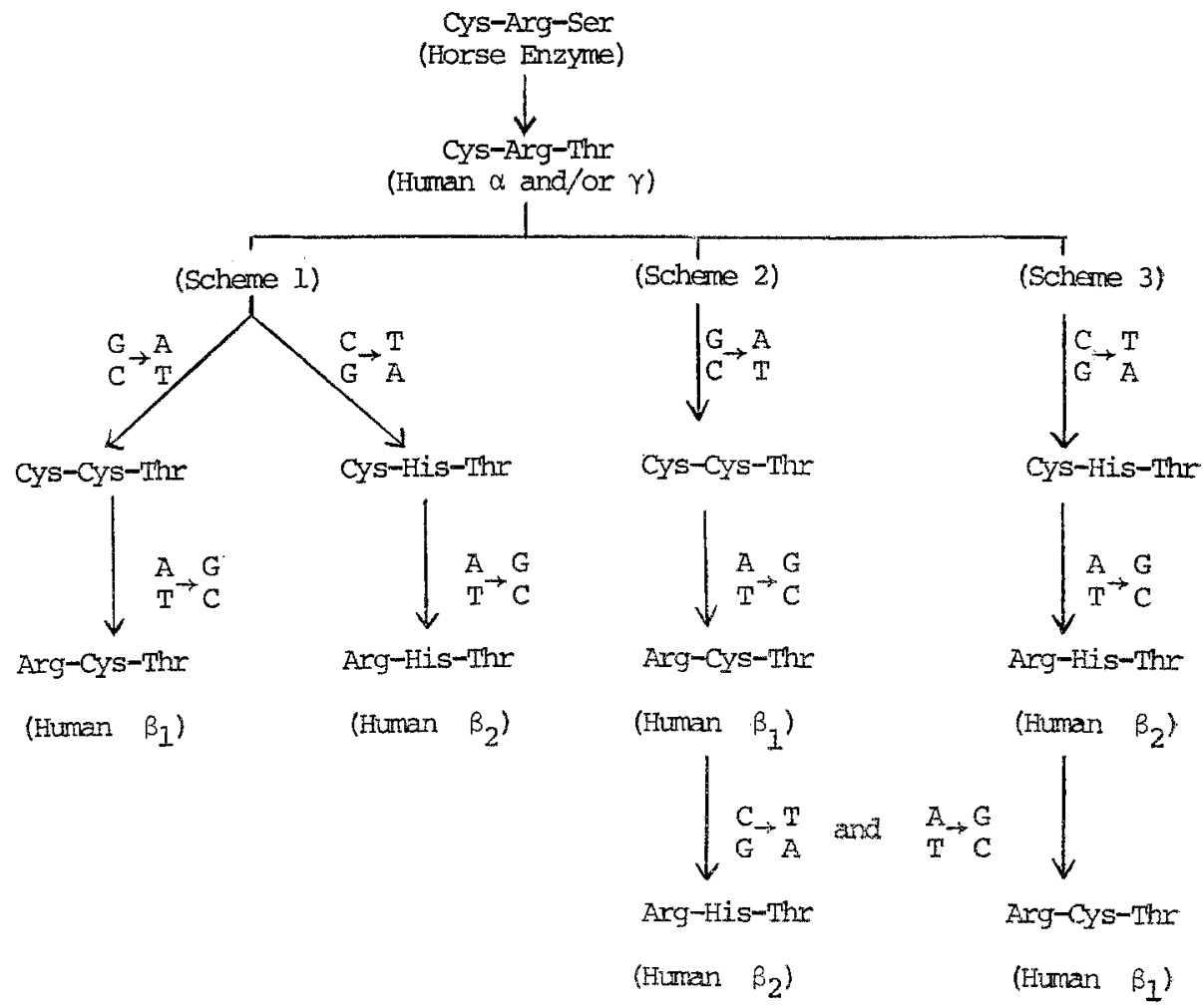

Fig. 7. Possible process of evolutional divergence of usual $\beta_{1} \beta_{1}$ and atypical $\beta_{2} \beta_{2}$ enzymes. Nucleotide base substitutions corresponding to the amino acid substitutions are also given.

produce $\beta_{2}$ from $\beta_{1}$ or vice-versa. In most cases, polymorphism is considered to have originated in a single base substitution at a given locus, or by non-reciprocal crossing-over. The human $\mathrm{ADH} \beta_{1}$ and $\beta_{2}$ are exceptional in this respect, and the underlying mechanism of the evolutional divergence is worth pursuing by examining the structure of $\mathrm{ADH}$ isozymes and the structure of ADH loci of various mammals. b) Aldehyde dehydrogenase. In comparison to ADH, our knowledge of ALDH is rather limited. ALDH from man and other mammals, is a tetrameric enzyme with molecular weight of about 230,000 .

Human liver ALDH-1, an isozyme with slower anodal electrophoretic mobility at neutral to basic $\mathrm{pH}$, has a low $K_{\mathrm{m}}$ for NAD and a high apparent $K_{\mathrm{m}}$ for acetaldehyde, and ALDH-2, an isozyme with faster anodal electrophoretic mobility, has. a high $K_{\mathrm{m}}$ for NAD and a low $K_{\mathrm{m}}$ for acetaldehyde (Greenfield and Pietruszko, 1977). Comparable isozymes exist in other mammals. Although no direct evidence has yet been presented, one can conclude, from the analogy of isozyme distribution in animal livers (Crow et al., 1974; Eckfeld and Yonetani, 1976; Kita- 
batake et al., 1981), that human ALDH-1 is of cytosolic and ALDH-2 is presumably of mitochondrial origin.

ALDH-1 and ALDH-2 were purified to homogeneity from an usual Caucasian liver. ALDH-1 was also purified from an atypical Japanese liver which had no ALDH-2 isozyme. A polyacrylamide gel electrophoresis in the presence of sodium dodecylsulfate, revealed that ALDH-1 consists of subunit with molecular weight of 56,500 , and ALDH-2 consists of subunit with molecular weight of 52,600. No common subunit exists in the two isozymes. Thus, ALDH-1 is presumably a homotetramer (aaaa), and ALDH-2 is another homotetramer (bbbb), although the heterotetramer model, i.e., ALDH-1 $\left(\mathrm{aa}^{\prime}\right)_{2}$ and/or ALDH-2 $\left(\mathrm{bb}^{\prime}\right)_{2}$, is not ruled out at the present time. Kinetic characteristics, i.e., pH-activity profile, $K_{\mathrm{m}}$ for NAD and for acetaldehyde, differ between ALDH-1 and ALDH-2. ALDH-1 had high affinity to NAD $\left(K_{\mathrm{m}}=5 \mu \mathrm{m}\right)$ and lower affinity to acetaldehyde $\left(K_{\mathrm{m}}=22 \mu \mathrm{M}\right)$, and ALDH2 had lower affinity to NAD $\left(K_{\mathrm{m}}=16 \mu \mathrm{M}\right)$, and higher affinity to acetaldehyde $\left(K_{\mathrm{m}}=\right.$ $3.5 \mu \mathrm{M})$ at $\mathrm{pH} 7.5$.

The kinetic data reported by Greenfield and Pietruszko (1977) differ from the present values, i.e., $K_{\mathrm{m}}$ for $\mathrm{NAD}=40 \mu \mathrm{M}, K_{\mathrm{m}}$ for acetaldehyde $=30 \mu \mathrm{M}$ in $\mathrm{ALDH}-1$, and $K_{\mathrm{m}}$ for $\mathrm{NAD}=70 \mu \mathrm{M}, K_{\mathrm{m}}$ for acetaldehyde $=3.0 \mu \mathrm{M}$ in $\mathrm{ALDH}-2$ at $\mathrm{pH} 7.0$. Since the enzyme did not exhibit the typical Michaelis-Menten kinetics, care should be taken in interpreting these kinetic data.

Rabbit antibody against ALDH-1 precipitated and neutralized not only ALDH1 but also ALDH-2. Antibody against ALDH-2 also reacted with both ALDH-1 and ALDH-2. Thus, structural homology exists between ALDH-1 and ALDH-2. Examination of the immunologically active components of liver extract by two dimensional starch gel electrophoresis followed by crossed immunoelectrophoresis revealed that an atypical Japanese liver, which had no ALDH-2 isozyme, contained an enzymatically inactive, but immunologically cross-reactive material (CRM) corresponding to ALDH-2, in addition to the active ALDH-1 isozyme (Fig. 8). ALDH-1 and CRM in the crude extract of an atypical Japanese liver were separated from each other by the treatment with 5'-AMP-Sepharose 4B, since ALDH-1 was absorbed with the affinity column, while CRM had no affinity to the NAD analogue, i.e., 5'-AMP.

The existence of CRM corresponding to ALDH-2 in an atypical liver leads us to conclude that the absence of ALDH-2 isozyme in about $50 \%$ of Japanese is not due to regulatory mutation, nonsense mutation, or gene deletion, as previously proposed (Teng, 1981), but it must be due to a structural mutation in a gene for ALDH-2 locus, resulting in the formation of enzymatically inactive abnormal protein. It would be interesting to investigate an exact amino acid substitution which caused the total loss of enzymatic activity in the mutant ALDH-2 protein. Thus, the structural requirement for ALDH activity will become apparent.

c) Relationships between the alcohol sensitivity and enzyme abnormalities. All investigators agree that a high frequency of acute alcohol intoxication was observed 


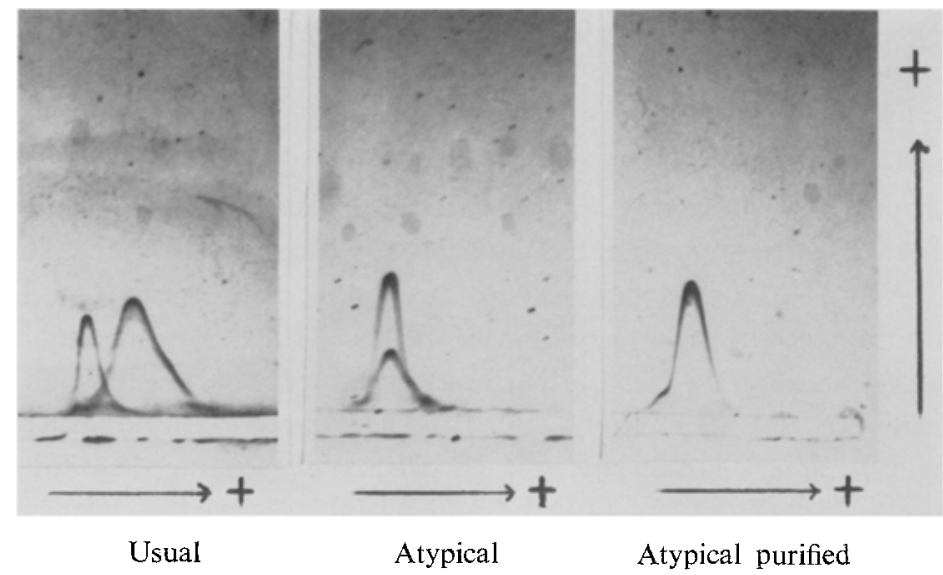

Fig. 8. Crossed immunoelectrophoresis of aldehyde dehydrogenase. 1st dimension (horizontal): Starch gel electrophoresis at pH 7.2. 2nd Dimension (vertical): Immunoelectrophoresis in agarose gel containing antibody against ALDH-2.

Samples: Left, partially purified enzyme preparation from an usual Caucasian liver; Middle, partially purified enzyme preparation from an atypical Japanese liver; Right, Enzyme preparation from an atypical Japanese liver purified by affinity chromatography with 2 -AMP-Sepharose. Note that an enzymatically inactive but immunologically active component is eliminated by the affinity chromatography.

Details were reported by Impraim et al. (1982).

in Orientals, but the figures differ among the investigators. Thus, about $83 \%$ of Wolff's Oriental population showed a flush response (Wolff, 1972, 1973); about 71\% in the study by Ewing et al. (1974), 50-60\% in studies by Wilson et al. (1978), and by Zeiner et al. (1979). The differences could be related to their sampling methods, i.e., random population versus adult "volunteers" with various degrees of predisposition to alcohol, and their test methods for the sensitivity. As far as sampling, Wolff's study (1972) using Oriental and Caucasian infants seems to be the most unbiased.

Blood alcohol level and rate of clearance after drinking a moderate amount of alcohol were examined by several investigators. Von Wartburg and Schürch (1968) observed significantly more rapid alcohol elimination in subjects with atypical ADH (i.e., $90 \mathrm{mg} / \mathrm{kg} / \mathrm{hr}$ in the normal and $141 \mathrm{mg} / \mathrm{kg} / \mathrm{hr}$ in the atypical), while Edwards and Evans (1967) found the faster elimination in only one of the three subjects with the atypical ADH. The type of ALDH of their subjects is unknown. In contrast, Fenna et al. (1971) reported that the rate of alcohol metabolism, after intravenous injection, was significantly higher in Caucasians than Mongoloids (i.e., $145 \mathrm{mg} / \mathrm{kg}$ / $\mathrm{hr}$ and $105 \mathrm{mg} / \mathrm{kg} / \mathrm{hr}$ respectively). The types of ADH and ALDH of these subjects are unknown. Mizoi et al. (1979) found no difference in either the maximum blood 
alcohol level (about $10 \mathrm{~mm}$ ) or the rate of elimination (about $100 \mathrm{mg} / \mathrm{kg} / \mathrm{hr}$ ) between alcohol flushing Japanese and non-flushing Japanese.

The data concerning acetaldehyde levels after alcohol intake have been widely ranged, because of difficulties in measuring the very volatile substance and the release of a bound form of acetaldehyde from red blood cells. The values given $(0.1-0.56 \mu \mathrm{g} / \mathrm{ml}$, i.e., average several $\mu \mathrm{M})$ by Lundquist and Westerfeld (1958), Truitt and Walch (1971) and Stowell et al. (1980), seem to be more reliable than the higher values $(9.3 \mu \mathrm{g} / \mathrm{ml}$ in Oriental males and $7.7 \mu \mathrm{g} / \mathrm{ml}$ in Caucasian males) reported by Ewing et al. (1974). Mizoi et al. (1979) reported that blood acetaldehyde reached an average $0.508 \mu \mathrm{g} / \mathrm{ml}$ (i.e., $11.5 \mu \mathrm{M}$ ) in alcohol sensitive Japanese and about 0.1 $\mu \mathrm{g} / \mathrm{ml}$ (i.e., $2.3 \mu \mathrm{M}$ ) in non-flushing Japanese. According to the recent study by Harada et al. (1981), the acetaldehyde level in blood reaches about $2 \mu \mathrm{M}$ in usual individuals, and about $35-40 \mu \mathrm{M}$ in alcohol sensitive subjects.

As previously suggested, either the atypical $\mathrm{ADH}_{2}{ }^{2}$ with high enzyme activity (Stamatoyannopoulos et al., 1975), or the absence of ALDH-2 isozyme (Goedde et al., 1979a) could account for an accumulation of acetaldehyde inducing alcohol intoxication. Alternatively, the combination of both ADH and ALDH abnormalities could be involved in the elevation of acetaldehyde level. Based on the available data on ethanol metabolism, one can attempt to evaluate these possibilities.

From the studies of perfused rat livers, more than $80 \%$ of ethanol administered at a moderate level is oxidized in liver. Most of the acetaldehyde formed is considered to be further oxidized to acetate in the liver (Williamson et al. 1969; Eriksson et al. 1975; Braggins et al. 1980), although Lindros et al. (1972) reported that more than $50 \%$ of acetaldehyde left from the liver unmetabolized under certain conditions. Multiple metabolic pathways are open to acetaldehyde (i.e., ALDH, aldehyde oxidase, xanthine oxidase, and a specific aldolase), but cytosolic ALDH-1 and mitochondrial ALDH-2 are considered to play a major role in acetaldehyde metabolism in the liver. Krebs concluded that rats' hepatic ADH activity is sufficiently high to establish near-equilibrium in reactants of the ADH system and the lactate dehydrogenase system (Krebs, 1966; Williamson et al., 1967). Thus, the rate of ethanol elimination depends on the concentration of acetaldehyde determined by the ADH step. His conclusion seems to be an over-simplified view, and an apparent near-equilibrium can only be observed in a particular condition (Veech et al., 1972; Braggins et al., 1980). The study by Braggins et al. (1980), suggests that the rat liver has a similar capacity for ethanol and acetaldehyde oxidation, and that changes in acetaldehyde level could occur by variations of ADH and/or ALDH activities. The situation is basically similar in man. It is known that a partial inhibition of ALDH by calcium carbimide or by disulfiram induces elevation of blood acetaldehyde levels and flushing reaction in man (Stowell et al., 1980). A partial inhibition of ADH by pyrazole attenuates acetaldehyde levels and symptoms caused by the ALDH inhibitor (Stowell et al., 1980).

As described above, the specific activity $\left(V_{\max }\right)$ of the pure $\mathrm{ADH}_{2}{ }^{2}$ is about 80 
times higher than the usual $\mathrm{ADH}_{2}{ }^{1}$ at the physiological $\mathrm{pH}$, and the $\mathrm{ADH}$ activity of crude homogenate of the liver with atypical ADH type is several times higher than that of the usual type liver. But the rate of ethanol oxidation in the atypical liver could be similar to that in the usual liver, since a near-equilibrium state is already established in the usual liver, and an additional several-fold increase in potential $\mathrm{ADH}$ activity in the atypical liver is not expected to induce substantial shift of the equilibrium state. Thus, the rate of ethanol elimination in the liver with atypical $\mathrm{ADH}$, is estimated to be only slightly higher than that in the usual liver, as actually observed (Von Wartburg and Schürch, 1968; Edwards and Evans, 1967). A decrease in ALDH activity in the atypical liver missing ALDH-2 isozyme could induce elevation of blood acetaldehyde level and flushing, as observed in the subjects administered with ALDH inhibitors such as disulfiram and calcium carbimide. These drugs reduce the rate of ethanol elimination in man (Stowell et al., 1980). By contrast, the rate of ethanol elimination is reported to be identical between alcohol flushing and non-flushing Japanese (Mizoi et al., 1979).

From these findings, a probable genetic cause of alcohol flushing in Orientals is a combination of atypical ADH and atypical ALDH. A higher ADH activity could elevate the acetaldehyde level which compensates for the decrease in ALDH activity and maintains the rate of ethanol elimination unchanged. The degree of acute alcohol intoxication is known to be heterogeneous (Mizoi et al., 1979). Subjects with atypical ADH type and atypical ALDH type are probably most sensitive, while subjects with usual ADH and atypical ALDH are moderately sensitive. Subjects with atypical ADH and usual ALDH could be only mildly sensitive to alcohol or similar to normal.

A more direct study for the elucidation of the relationships between the dehydrogenase abnormalities and alcohol sensitivity is obviously the dehydrogenase typing of alcohol sensitive and insensitive individuals. Since it is difficult to obtain biopsy liver samples Goedde et al. $(1979 \mathrm{~b}, 1980)$ tried to distinguish ADH and ALDH types using fibroblast cultures and hair roots. The atypical $\mathrm{ADH}-\beta_{2} \beta_{2}$ isozyme is more basic than the usual $\beta_{1} \beta_{1}$ and moves faster towards the cathode in starch gel electrophoresis (Figs. 1 and 2), while the opposite isoelectric focussing patterns were observed in their typing (Goedde et al., 1979b). Since the structural abnormality of the atypical $\beta_{2} \beta_{2}$ is a single amino acid substitution from $\mathrm{Cys} \rightarrow \mathrm{His}$ at position 47, the atypical enzyme should be more basic (as it is found in starch gel electrophoresis), not more acidic (as they observed in isoelectric focussing). Besides the liver ALDH-1 and ALDH-2 isozymes, other tissues contained several different ALDH isozymes which seem to exhibit more or less tissue specific expressions (Harada et al., 1979). Origin and assignment of these isozyme components from liver, fibroblasts and hair roots in isoelectric focussing, require further careful examination. 


\section{CONCLUSION}

Our studies clarified some genetic and molecular differences in alcohol metabolism between Orientals and Caucasians. Approximately $90 \%$ of Orientals have the atypical $\mathrm{ADH}_{2}{ }^{2}\left(\beta_{2} \beta_{2}\right)$ isozyme, while virtually all Caucasians have the usual isozyme. The molecular difference between the usual and atypical enzymes is a single amino acid substitution $\mathrm{Cys} \rightarrow \mathrm{His}$ at position 47 . Approximately $50 \%$ of Orientals do not have mitochondrial ALDH-2 isozyme, while all Caucasians have this isozyme. The absence of ALDH-2 in Orientals is not due to gene deletion, regulatory mutation or nonsense mutation. Structural mutation had occurred in ALDH-2 locus, since an enzymatically inactive but immunologically cross-reaction material is produced in the atypical Orientals. Exact structural defect in the atypical protein remains to be elucidated.

The genetic polymorphism of ADH and that of ALDH is associated with wide differences in frequencies between Orientals and Caucasians. The mechanism of evolutional divergence of the usual and atypical enzymes would be an interesting subject to be explored. Despite the rapid development of human chromosome mapping, gene loci for ADH isozymes and ALDH isozymes have not yet been known. The study of structure of loci for ADH and ALDH in Orientals and Caucasians would contribute towards elucidating the evolutional process in these populations.

At the present time, there is no sufficient data for distinguishing between the following possibilities for the racial differences in alcohol sensitivity. This could be related to ADH abnormality, absence of ALDH-2 isozyme, or more likely a combination of these two factors. Besides the abnormalities of ADH and ALDH, the involvement of other genetic factors is not ruled out. Approximately $90 \%$ of Orientals have the atypical $\mathrm{ADH}_{2}{ }^{2}$, and therefore most of the Orientals with atypical ALDH type are also atypical in ADH. Although the hair root analysis by Goedde et al. (1980) suggested that the atypical ALDH, not atypical ADH, would be related to alcohol flushing, the number of subjects with crucial types were very limited and the typing was not absolutely certain. Family pedigree analysis of alcohol sensitivity and the unequivocal dehydrogenase typing, possibly using biopsy or autopsy livers, could solve the problem. Considering the availability of samples with various dehydrogenase types, such study may be more feasible in Japan or other Oriental countries rather than in Occidental countries.

There is a paradox between alcohol sensitivity (i.e., acute alcohol intoxication) and alcoholism. Both Orientals (Japanese, Chinese, Korean) and American Indians are of Mongoloid origin and the frequency of alcohol sensitivity is equally high in these populations. The incidence of alcoholism in Orientals is lower than that in Caucasians. In contrast, alcoholism is a known social problem among the American Indians. The genetic difference in alcohol metabolism due to atypical $\mathrm{ADH}$ and/or ALDH would have a dual effect. The acute intoxication effect might 
discourage individuals from drinking, thus decreasing the alcoholism. On the other hand, if individuals with the abnormality persist in drinking alcohol for psychological and/or environmental reasons, prolonged elevation of acetaldehyde could lead to a formation of toxic materials related to alcohol addiction (Davis and Walsh, 1970). Several investigators have suggested that a hepatic ethanol-inducible microsomal alcohol dehydrogenase and $\mathrm{ADH}-\pi$ play a substantial role in the handling of a high level of alcohol over a long period of time (Orme-Johnson and Ziegler, 1965; Lieber and DeCarli, 1968; Li et al., 1977). Whether or not genetic differences of microsomal and $\mathrm{ADH}-\pi$ exist between Orientals and American Indians, remains unanswered.

Another interesting problem of alcohol sensitivity is that a high frequency of intoxication is observed among diabetics (Caucasians) after chloropropamide administration. Originally, Leslie and Pyke $(1978,1979)$ reported that this high frequency was observed only in maturity-onset (type 2) diabetics and their relatives, but not in juvenile-onset (type 1) diabetics and control subjects. Their observations suggested that chloropropamide-induced alcohol flushing is a dominantly inherited trait associated with type 2 diabetes. However, a recent study disputes these previous reports, i.e., diabetics, regardless of type, exhibited chloropropamide-induced alcohol sensitivity with high frequency (Dreyer et al., 1980). Although abnormalities of $\mathrm{ADH}$ and $\mathrm{ALDH}$ in diabetics have not yet been studied, the high frequency of chloropropamide-induced alcohol flushing is likely to be due to a secondary consequence of diabetes.

Acknowledgement This study was supported by the U.S. Public Health Service Grant HL15125. The author wishes to thank Drs. I-Y. Huang, C.C. Impraim, and J.F. Lee for their contribution in this study, and to Mr. G. Wang for technical assistance. The author also thanks Dr. A. Hayashi, Osaka University School of Medicine, for providing autopsy Japanese livers.

\section{REFERENCES}

Borg, S., and Kvande, H. 1981. Control norepinephrine metabolism during alcohol intoxication in addicts and healthy volunteers. Science 213: 1135-1137.

Braggins, T.J., Crow, K.E., and Bott, R.D. 1980. Acetaldehyde and acetate production during ethanol metabolism in perfused rat liver. Adv. Exptl. Med. Biol. 132: 441-449.

Crow, K., Kitson, T.M., MacGibbon, A.K.H., and Bott, R.D. 1974. Intracellular localization and properties of aldehyde dehydrogenase from sheep liver. Biochim. Biophys. Acta 350: 121128.

Dahl, K.H., and McKinley-McKee, J.S. 1980. Phosphate binding to liver alcohol dehydrogenase studied by the rate of alkylation with affinity labels. Eur. J. Biochem. 103: 47-51.

Davis, V.E., and Walsh, J.J. 1970. Alcohol, amines, and alkaloids: A possible biochemical basis for alcohol addiction. Science 167: 1005-1007.

Dreyer, M., Kühnau, J., and Rüdiger, M.W. 1980. Chlorpropamide-alcohol flushing is not useful for individual genetic counseling of diabetic patients. Clin. Genet. 18: 189-190.

Duritz, G., and Truitt, E.B. 1966. Importance of acetaldehyde in the action of ethanol on brain norepinephrine and 5-hydroxytryptamine. Biochem. Pharmacol. 15: 711-721. 
Eckfeld, J.H., and Yonetani, T. 1976. Subcellular localization of the $F_{1}$ and $F_{2}$ isozymes of horse liver aldehyde dehydrogenase. Arch. Biochem. Biophys. 175: 717-722.

Edwards, J.A., and Evans, D.A.P. 1967. Ethanol metabolism in subjects possessing typical and atypical liver alcohol dehydrogenase. Clin. Pharmacol. Therapeu. 8: 824-829.

Eklund, H., Nordström, B., Zeppezauer, E., Söderlund, G., Ohlsson, I., Boiwe, T., Söderberg, B-O., Tapia, O., and Brändén, C-I. 1976. Three-dimensional structure of horse liver alcohol dehydrogenase at $2.4 \AA$ resolution. J. Mol. Biol. 102: 27-59.

Eriksson, C.J.P., Marselos, M., and Koivula, T. 1975. The role of cytosolic rat liver aldehyde dehydrogenase in the oxidation of acetaldehyde during ethanol metabolism in vivo. Biochem. $J$. 152: 709-712.

Ewing, J.A., Rouse, B.A., and Pellizzari, E.D. 1974. Alcohol sensitivity and ethnic background. Am. J. Psychiatr. 131: 206-210.

Fenna, D., Mix, L., Schaefer, O., and Gilbert, J.A.L. 1971. Ethanol metabolism in various racial groups. Can. Med. Assoc. J. 105: 472-475.

Goedde, H.W., Harada, S., and Agarwal, D.P. 1979a. Racial differences in alcohol sensitivity: A new hypothesis. Hum. Genet. $51: 331-334$.

Goedde, H.W., Agarwal, D.P., and Harada, S. 1979b. Alcohol metabolizing enzymes: Studies of isozymes in human biopsies and cultured fibroblasts. Clin. Genet. 16: 29-33.

Goedde, H.W., Agarwal, D.P., and Harada, S. 1980. Genetic studies on alcohol-metabolizing enzymes: Detection of isozymes in human hair roots. Enzyme 25: 281-286.

Greenfield, N.J., and Pietruszko, R. 1977. Two aldehyde dehydrogenase from human liver: Isolation via affinity chronatography and characterization of isozymes. Biochint. Biophys. Acta 483: $35-45$.

Harada, S., Agarwal, D.P., and Goedde, H.W. 1979. Aldehyde dehydrogenase in human blood and its possible role in alcohol metabolism. Report 8 th Int. Congr. Forensic Blood Grouping. London. pp. 497-502.

Harada, S., Agarwa1, D.P., and Goedde, H.W. 1981. Acetaldehyde metabolism and polymorphism of aldehyde dehydrogenase in Japanese. Abstract, 6th Int. Congr. Hum. Genet. 103.

Impraim, C.C., Wang, G, and Yoshida, A. 1982. Structural mutation in a major human aldehyde dehydrogenase gene results in loss of enzyme activity. Am. J. Hum. Genet. in press.

Jörnvall, H. 1970. Horse liver alcohol dehydrogenase. The primary structure of the protein chain of the ethanol-active isozyme. Eur. J. Biochem. 16: 25-40.

Jörnvall, H., and Pietruszko, R. 1972. Structural studies of alcohol dehydrogenase from human liver. Eur. J. Biochem. 25: 283-290.

Kitabatake, N., Sasaki, R., and Chiba, H. 1981. Localization of bovine liver aldehyde dehydrogenase isozymes and their immunological properties. J. Biochem. 89: 1223-1229.

Krebs, H.A. 1966. The role of equilibria in the regulation of metabolism. in Current Topics in Cellular Regulation I, Horecker, B.L., and Stadtman, E.R. eds., pp. 45-55. Academic Press, New York.

Leslie, R.D.G., and Pyke, D.A. 1978. Chlorpropamide-alcohol flushing: a dominantly inherited trait associated with diabetes. Brit. Med. J. Dec. 2: 1519-1521.

Leslie, R.D.G., and Pyke, D.A. 1979. Sensitivity to enkephalin as a cause of non-insulin dependent diabetes. Lancet Feb.: 341-343.

Li, T-K., Bosron, W.F., Dafeldecker, W.P., Lange, L.G., and Vallee, B.L. 1977. Isolation of $\pi^{-}$ alcohol dehydrogenase of human liver: Is it a determinant of alcoholism? Proc. Natl. Acad. Sci. USA. 74: 4378-4381.

Lieber, C.S., and DeCarli, L.M. 1968. Ethanol oxidation by hepatic microsomes: Adaptive increase after ethanol feeding. Science 162: 197-198.

Lindros, K.O., Vihma, R., and Forsander, O.A. 1972. Utilization and metabolic effect of acetaldehyde and ethanol in the perfused rat liver. Biochem. J. 126: 945-952. 
Lundquist, F., and Westerfeld, W.W. 1958. The kinetics of alcohol elimination in man. Acta Pharmacol. Toxicol. 14: 265-289.

Mizoi, Y., Ijiri, I., Tatsuno, Y., Kijima, T., Fujiwara, S., and Adachi, J. 1979. Relationship between facial flushing and blood acetaldehyde levels after alcohol intake. Pharm. Biochem. Behav. 10: $303-311$.

Orme-Johnson, W.H., and Ziegler, D.M. 1965. Alcohol mixed function oxidase activity of mammalian liver microsomes. Biochem. Biophys. Res. Commun. 21: 78-82.

Smith, M., Hopkinson, D.A., and Harris, H. 1971. Developmental changes and polymorphism of human liver alcohol dehydrogenase. Am. Hum. Genet. 34: 251-261.

Smith, M., Hopkinson, D.A., and Harris, H. 1972. Alcohol dehydrogenase isozymes in adult human stomach and liver: Evidence for activity of the $A D H_{3}$ locus. Ann. Hum. Genet. 35: 243-253.

Smith, M., Hopkinson, D.A., and Harris, H. 1973. Studies on the subunit structure and molecular size of the human alcohol dehydrogenase isozymes determined by the different loci, $A D H_{1}$, $\mathrm{ADH}_{2}$, and $\mathrm{ADH}_{3}$. Ann. Hum. Genet. 36: 401-414.

Stamatoyannopoulos, G., Chen, S-H., and Fukui, M. 1975. Liver alcohol dehydrogenase in Japanese: High population frequency of atypical form and its possible role in alcohol sensitivity. Am. J. Hum. Genet. 27: 789-796.

Stowell, A., Hillbom, M., Salaspuro, M., and Lindros, K.O. 1980. Low acetaldehyde levels in blood, breath and cerebrospinal fluid of intoxicated humans as assayed by improved methods. Adv. Exptl. Med. Biol. 132: 635-645.

Teng, Y-S. 1981. Human liver aldehyde dehydrogenase in Chinese and Asiatic Indians: Gene deletion and its possible implication in alcohol metabolism. Biochem. Genet. 19: 107-113.

Truitt, E.B., and Walsh, M.J. 1971. The role of acetaldehyde in the actions of ethanol. In The Biology of Alcoholism, Vol. 1. Biochemistry, Kissin, B. and Begleiter, H., eds., New York Plenum Pub., New York

Veech, R.L., Guynn, R., and Veloso, D. 1972. The time-course of the effects of ethanol on the redox and phosphorylation states of rat liver. Biochem. J. 127: 387-397.

Von Wartburg, J.P., and Schürch, P.M. 1968. Atypical human liver alcohol dehydrogenase. Ann. New York Acad. Sci. 151: 936-946.

Williamson, D.H., Lund, P., and Krebs, H.A. 1967. The redox state of free nicotinamide-adenine dinucleotide in the cytoplasm and mitochondria of rat liver. Biochem. J. 103: 514-527.

Williamson, J.R., Scholz, R., Browning, E.T., Thurman, R.G., and Fukami, M.H. 1969. Metabolic effects of ethanol in perfused rat liver. J. Biol. Chem. 244: 5044-5054.

Wilson, J.R., McClearn, G.E., and Johnson, R.C., 1978. Ethnic variation in the use and effects of alcohol. Drug Alcohol Depend. 3: 147-151.

Wolff, P.C. 1972. Ethnic differences in alcohol sensitivity. Science 175: 449-450.

Wolff, P.C. 1973. Vasomotor sensitivity to alcohol in diverse Mongoloid populations. Am. J. Hum. Genet. 25: 193-199.

Yoshida, A., Impraim, C.C., and Huang, I-Y. 1981. Enzymatic and structural differences between usual and atypical human liver alcohol dehydrogenases. J. Biol. Chem. 256: 12430-12436.

Zeiner, A.R., Paredes, A., and Dix Christensen, H. 1979. The role of acetaldehyde in mediating reactivity to an acute dose of ethanol among different racial groups. Clin. Expt. Res. 3: 11-18. 